\title{
Measurement of $f$ orbital hybridization in rare earths through electric dipole-octupole interference in X-ray Absorption Spectroscopy
}

\author{
Amélie Juhin, ${ }^{1, *}$ Stephen P. Collins, ${ }^{2}$ Yves Joly, ${ }^{3}$ Maria Diaz-Lopez,${ }^{4,2,3}$ \\ Kristina Kvashnina, ${ }^{5}$ Pieter Glatzel, ${ }^{5}$ Christian Brouder, ${ }^{1}$ and Frank de Groot $^{6}$ \\ ${ }^{1}$ Institut de Minéralogie, de Physique des Matériaux et de Cosmochimie (IMPMC), Sorbonne Université, \\ UMR CNRS 7590, Université Pierre et Marie Curie, 4 place Jussieu, 75052 Paris Cedex 05, France \\ ${ }^{2}$ Diamond Light Source Ltd., Diamond House, Harwell Science and Innovation Campus, Didcot OX11 ODE, UK \\ ${ }^{3}$ Univ. Grenoble Alpes, CNRS, Grenoble INP, Institut Néel, 38000 Grenoble France \\ ${ }^{4}$ ISIS Facility, STFC Rutherford Appleton Laboratory, Didcot, Oxfordshire, OX11 0QX, UK \\ ${ }^{5}$ European Synchrotron Radiation Facility, 25 Avenue des Martyrs, 38042 Grenoble Cedex, France \\ ${ }^{6}$ Debye Institute of Nanomaterials Science, Utrecht University, 3584 CG Utrecht, Netherlands
}

This work provides a direct route to measure the degree of hybridization of $f$ states in rare earths. The interference between electric dipole and octupole transitions is measured at the $L_{1}$ edge of Gd in $\mathrm{Gd}_{3} \mathrm{Ga}_{5} \mathrm{O}_{12}$ using X-ray Natural Linear Dichroism (XNLD) and high energy resolution fluorescence detection. The Gd $4 f-6 p$ admixture is quantified through the integral of the dipoleoctupole XNLD using a new sum rule easily applicable to experimental data. The mixing of the Gd valence states with the $\mathrm{O}$ ligand orbitals, calculated from first-principles, reveals that despite their localized character, the Gd $4 f$ orbitals mix with the $\mathrm{O} 2 p$ and $2 s$ orbitals with an antibonding and bonding character, respectively.

PACS numbers: 78.70.Dm,71.15.Mb,71.20.-b,71.20.Eh

Lanthanide and actinide based materials have been increasingly used in diverse industrial processes such as catalysis, energy production, medicine, production of alloys, magnets and lighting materials [1]. They also build a fascinating class of materials for physicists due to their peculiar electronic structure that produces mixed valency, heavy fermion or Kondo like behaviours. Despite considerable progress achieved recently, the deep understanding of the ground state (in particular, $f$ orbital occupancy and the role of $f / d$ orbitals in bonding), remains a challenge for both experiment and theory [2]. The $4 f$ orbitals are often viewed as core-like states and weakly involved in bonding, but recent studies of $4 f$ based compounds showing an unexpected $4 f$ and $5 d$ orbital contribution to chemical bonding have questioned this picture [3]. For actinides (especially, early elements), the participation to bonding of $5 f / 6 d$ orbitals and of pseudo-core $6 s / 6 p$ orbitals has been one of the most longstanding debates $[4,5]$. In this work, we present a novel experimental approach to quantify directly the hybridization of $f$ orbitals in the rare earth ground state: this information on the electronic structure, inaccessible so far, is expected to benchmark models of their electronic structure and to revive the discussion on the localization of $f$ states in rare earths, regarding their implication in e.g. chemical reactivity, ultra-fast demagnetization and luminescent properties.

Our approach is based on X-ray Absorption Spectroscopy (XAS) that has been widely used as an elementspecific probe of the electronic structure [6]. XAS measures the transition probability for a core electron of an absorbing ion to be promoted into unoccupied electronic states, which is mainly achieved by electric dipole
$(\Delta \ell=1) E 1$ transitions and in second order by electric quadrupole $(\Delta \ell=2) E 2$ transitions. XAS provides insight into the local electronic structure of the absorber, including valency, ligand-metal covalency or onsite mixing of atomic orbitals, as it has been demonstrated in rare earth compounds using both ligand and rare earth absorption edges (e.g. [7-15]). Over the years, the technique has been popularized under different forms, amongst which, X-ray Magnetic Circular and Linear Dichroism (XMCD [16, 17] and XMLD [18]), Xray Natural Circular and Linear Dichroism (XNCD [19] and XNLD [20]), exploiting the dependence of XAS upon the polarization state and/or the direction of the incident $\mathrm{x}$-rays to reveal anisotropy in charge, orbital or spin distribution, or the mixing between electronic states with different parity.

Most of the XAS experiments probing empty $f$ states in rare earths have been performed at the $M_{4,5} / N_{4,5}$ and $L_{2,3} / M_{2,3}$ edges using dipole and quadrupole transitions, with a significant improvement in experimental resolution achieved recently thanks to high energy resolution fluorescence detection (HERFD) [21, 22]. In this paper, we purposely choose the $L_{1}$ edge because the deep, spinorbit free $2 s$ corehole allows discarding some of the strong intraatomic multielectronic effects that complicate the interpretation of other edges: $f$ states are then only accessible by electric octupole $E 3$ transitions $(\Delta \ell=3)$, a contribution that has always been dismissed in the past due to the expected weak intensity [20] but that finds a renewed interest [23]. Here, we demonstrate the possibility to directly probe empty $f$ states at the $L_{1}$ edge through the interference between $E 1$ and $E 3$ transitions, by measuring XNLD with linearly polarized x-rays, using 
HERFD detection, and taking advantage of the increase in x-ray photon flux and energy resolution available on third-generation synchrotron sources.

We consider the case of $\mathrm{Gd}$ in $\mathrm{Gd}_{3} \mathrm{Ga}_{5} \mathrm{O}_{12}$ (GGG) for two reasons: ( $i$ ) as $4 f$ states are expected to be highly localized, GGG represents an excellent test case to demonstrate the sensitivity of our approach, (ii) the cubic crystal structure, as will be shown, allows directly measuring the degree of hybridization between the Gd empty $f$ and $p$ states (i.e., orbitals with same parity) by integration of the XNLD signal. The ground state electronic structure is then analyzed using first-principle calculations to quantify the hybridization of the Gd orbitals with the $\mathrm{O}$ ligand orbitals and the Gd $5 d$ crystal field splitting.

Experiments. A very high quality single crystal of GGG cut along (110) was positioned at $45^{\circ}$ with respect to the incident beam with linear horizontal polarization [24]. It was rotated clockwise about the [110] axis by an angle $\alpha$ defined such that for $\alpha=90^{\circ}$, the [001] axis of the crystal is vertical (Fig. 1). A $2 s 5 p$ RIXS plane was first measured at the Gd edge (resonant $2 s$ excitation followed by $5 p \rightarrow 2 s$ emission). Two series of HERFD-XAS spectra were collected at the $L_{1}$ edge first by setting the emission energy to $\mathrm{E}_{e m 1}=8347.6 \mathrm{eV}$ and then to $\mathrm{E}_{e m 2}=8353.0 \mathrm{eV}$. For each series, $\alpha$ was varied by steps of $10^{\circ}$ between $0^{\circ}$ and $360^{\circ}$. Measurements were performed at ID26 beamline (ESRF) using the undulator fundamental, $\mathrm{Si}(311)$ crystals to select the incident energy and four $\mathrm{Si}(511)$ crystals arranged in a vertical Rowland geometry $(R \approx 1 \mathrm{~m})$ to detect the inelastically scattered photons, with a combined resolution of $\Delta E \approx 0.5 \mathrm{eV}$.

First-principle simulations. XAS spectra were computed from first-principles using FDMNES [25]. A selfconsistent calculation including relativistic effects was performed in the Finite Difference approach using a full screening of the core hole on a $7.0 \AA$ cluster of GGG [26]. Spectra were convolved with a Lorentzian accounting for the $2 s$ core-hole lifetime. The cross-section was normalized and rescaled in energy with respect to experiment. From the ground state electronic structure, partial Density Of States (DOS) and Crystal Overlap Orbital Population (COOP) were extracted in order to analyze the Gd-O bonding. The COOP was calculated as [27]:

$\operatorname{COOP}_{\ell_{a} \ell_{b}}=\sum_{m_{a}, m_{b}, f} a_{\ell_{a} m_{a}}^{f} a_{\ell_{b} m_{b}}^{f} \int \varphi_{\ell_{a} m_{a}}(\mathbf{r}) \varphi_{\ell_{b} m_{b}}(\mathbf{r}) d \mathbf{r}$ where $\varphi_{\ell m}(\mathbf{r})$ are the normalized orbitals of atoms $a$ and $b$, and $a_{\ell m}^{f}$ the amplitudes given by the DFT calculation for all states $f$. The spin index is omitted for simplicity. The integral is performed in a sphere of radius equal to the half sum of atomic radius of both atoms and centered between them. Covalency between both neighboring atoms is therefore quantified, its positive/negative sign indicating respectively a bonding/anti-bonding char-

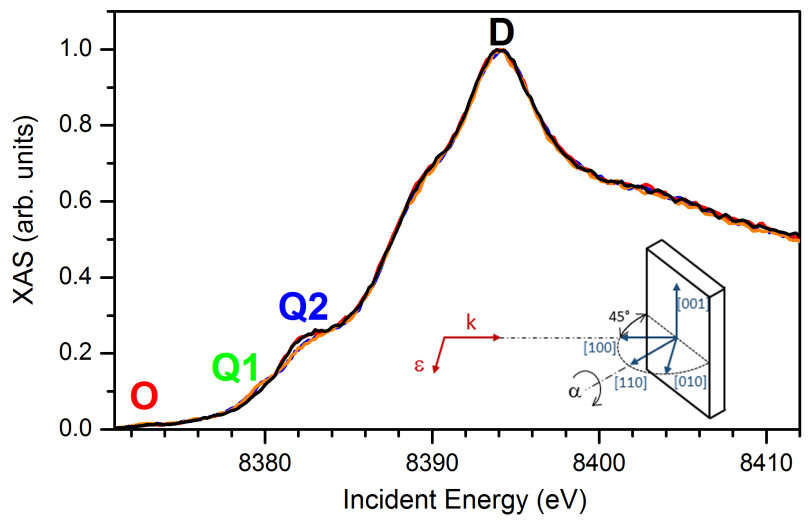

FIG. 1: Gd $L_{1}$ edge HERFD-XAS spectra measured in GGG using the $5 p \rightarrow 2 s$ x-ray emission channel for different rotation angles: $\alpha=0^{\circ}$ (orange), $90^{\circ}$ (black), $180^{\circ}$ (blue) and $270^{\circ}$ (red). Inset : configuration for $\alpha=90^{\circ}$.

acter.

$X$-ray Natural Linear Dichroism in GGG. The XAS cross-section is written as :

$$
\begin{aligned}
\sigma(\hat{\boldsymbol{\varepsilon}}, \hat{\mathbf{k}})=4 \pi^{2} \alpha_{0} \hbar \omega \sum_{F} & |\langle F|E 1+E 2+E 3| I\rangle|^{2} \\
& \times \delta\left(E_{F}-E_{I}-\hbar \omega\right),
\end{aligned}
$$

where $\alpha_{0}$ is the fine structure constant, $\hbar \omega$ is the photon incident energy, $|I\rangle$ and $|F\rangle$ are the initial and final states with respective energies $E_{I}$ and $E_{F} . E 1=\hat{\varepsilon} \cdot \mathbf{r}$ is the electric dipole operator, $E 2=\frac{i}{2} \hat{\varepsilon} \cdot \mathbf{r} \hat{\mathbf{k}} \cdot \mathbf{r}$ the electric quadrupole operator and $E 3=-\frac{1}{6} \hat{\varepsilon} \cdot \mathbf{r}(\hat{\mathbf{k}} \cdot \mathbf{r})^{2}$ the electric octupole operator [23]. The contribution of the magnetic dipole operator is negligible in the $\mathrm{x}$-ray range ([20], [36]) and we also neglect that of the spin-position operator, which is of relativistic origin and sizeable only in XMCD [28]. The relative strength of $E 2$ and $E 3$ with respect to $E 1$ can be estimated by evaluating these operators at the $2 s$ core-state radius $r_{2 s}$ of $\mathrm{Gd}\left(0.03189 a_{0}\right.$, deduced from the effective nuclear charge) using the $L_{1}$ edge energy for $\operatorname{Gd}(8.395 \mathrm{keV}): \frac{E 2}{E 1} \approx \frac{k r_{2 s}}{2}=3.610^{-2}$ and $\frac{E 3}{E 1} \approx \frac{\left(k r_{2 s}\right)^{2}}{6}=8.510^{-4}$.

Expansion of the squared matrix element in Eq.1 leads to six terms, three of which are squared elements: $\sigma(E 1, E 1)$ (dipole), $\sigma(E 2, E 2)$ (quadrupole) and $\sigma(E 3, E 3)$ (octupole). The other three terms are sums of two cross-products of matrix elements involving different operators: $\sigma(E m, E n)$ with $m \neq n$ [37]. Relatively to the dominant dipole contribution, quadrupole and octupole terms are smaller by a factor of roughly 2. $10^{-3}$ and $7 \cdot 10^{-7}$, respectively. The contributions of the cross-terms $\sigma(E 1, E 2), \sigma(E 1, E 3)$ and $\sigma(E 2, E 3)$ are respectively $4.10^{-2}, 8 \cdot 10^{-4}$ and $4 \cdot 10^{-5}$ with respect to the dipole. Since GGG crystallizes in a cubic structure, the dipole is isotropic [29] (i.e. it has no angular dependence) and $|I\rangle$ and $|F\rangle$ are parity invariant: this 


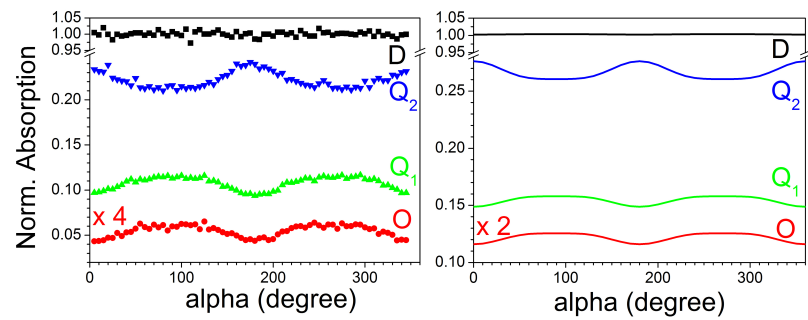

FIG. 2: Dependence of experimental (left) and theoretical (right) XAS intensity with respect to the rotation angle measured at four different values of incident energy : $8394.5 \mathrm{eV}$ (D), $8382.0 \mathrm{eV}$ (Q2), $8379.8 \mathrm{eV}$ (Q1) and $8373.0 \mathrm{eV}(\mathrm{O})$.

implies that both $\sigma_{\text {cube }}(E 1, E 2)$ and $\sigma_{\text {cube }}(E 2, E 3)$ are zero, which leaves $\sigma(E 1, E 3)$ as the only cross-term, referred to in the following as the interference term. For a cubic crystal, the quadrupole is expressed as the sum of one isotropic part $\sigma_{\text {cube }}^{\text {iso }}(E 2, E 2)$ and one anisotropic part $\sigma_{\text {cube }}^{\text {dichro }}(E 2, E 2)[30]$. For the present experiment, the quadrupole anisotropic part, which gives rise to quadrupole XNLD, has an angular dependence expressed as $(-19+60 \cos 2 \alpha+15 \cos 4 \alpha)$ where $\alpha$ is the rotation angle [31]. We have used spherical tensors to predict the angular dependence of the interference term in the case of a cubic crystal and for the present experiment (Suppl. Inf. II [32]). It is found that the anisotropic part of the interference term $\sigma_{\text {cube }}^{\text {dichro }}(E 1, E 3)$ has the same angular dependence as the quadrupole.

Origin of XNLD at the $L_{1}$ edge in $G G G$. Figure 1 shows the HERFD-XAS spectra measured at the Gd $L_{1}$ edge for four values of the rotation angle $\left(\alpha=0^{\circ}, 90^{\circ}\right.$, $180^{\circ}$ and $270^{\circ}$ ). Four spectral features are higlighted, labeled respectively $\mathbf{D}$ (main edge), Q1 and Q2 (rising edge), and $\mathbf{O}$ (pre-edge). A first tentative assignement can be made based on the relative energy position of the levels in atomic $\mathrm{Gd}$, which suggests that peak $\mathbf{D}$ is likely due to transitions from $2 s$ to $6 p$ empty states, that peaks Q1 and Q2 may involve transitions to the empty $5 d$ states, while peak $\mathbf{O}$ could be due to transitions involving empty $4 f$ states. Nevertheless, the nature of the empty states involved in the transitions (especially for peak $\mathbf{O}$ ) will be further inquired in the following using XNLD and first-principle calculations. Note the near perfect match of pairs of spectra rotated by $180^{\circ}$, which suggests at this point a $\pi$-periodicity for the total cross-section.

Figure 2 (left) presents the angular dependence of the experimental spectra as a function of rotation angle, when the incident energy is successively fixed at the energy position for each of the abovementioned four spectral features. For peak $\mathbf{D}$, the intensity is constant with $\alpha$, which is consistent with the dominant isotropic dipole character of the transitions $(2 s \rightarrow 6 p)$ at this energy. The dependence for peaks $\mathbf{Q 1}$ and Q2 is $\pi$-periodic in $\alpha$, which is compatible with the predicted dependence in $(-4 \cos 2 \alpha+\cos 4 \alpha)$. The angular dependence for peak
O follows that of peak Q1, which, at this point does not allow to interpret its origin. The angular dependence obtained from first-principle calculations (Fig. 2 right) is in excellent agreement with the experiment. Although the calculated intensity of peaks $\mathbf{Q 1}$ and $\mathbf{O}$ is overestimated, their $\alpha$-dependence is nicely reproduced, which allows futher quantitative interpretation regarding the nature of the states involved in the transitions.

In Fig. 3a the total XAS cross-section calculated for $\alpha=90^{\circ}$ are shown, together with the contributions of the different terms: dipole, quadrupole, octupole, and interference term. It is clear that the dipole is the main contribution to intensity in the measured spectral range, which indicates that empty $p$ states are involved in features $\mathbf{O}$, Q1, Q2 and D. The quadrupole contributes only to the rising edge, i.e. close to peaks $\mathbf{Q 1}$ and $\mathbf{Q} 2$, which is consistent with the expected transitions to empty $5 d$ states. In the region of peak $\mathbf{O}$, the octupole and the interference term contribute weakly to the absorption cross-section, which is dominated by the dipole (note that the interference term can be negative because it is not a squared matrix element). This indicates that at this energy, empty $4 f$ states hybridized with empty $6 p$ states are reached (see also the calculated DOS in Suppl. Inf. I [32]). Quantifying the degree of admixture between $6 p$ and $4 f$ states that are of same parity, which is allowed in cubic crystals (as opposite to states having different parities), will be discussed in the next subsection.

Figure $3 \mathrm{~b}$ shows the theoretical angular dependence calculated at the energy of peak $\mathbf{O}$ with the contribution of the different terms. Although the dipole is the dominant contribution to the XAS intensity, it has no dependence in $\alpha$ and thus does not contribute to XNLD at this energy. The quadrupole has almost zero intensity because the contribution of $5 d$ states to the DOS is small at this energy (Suppl. Inf. I [32]), and the octupole shows no dependence in $\alpha$. In the end, the XNLD calculated for peak $\mathbf{O}$ is solely due to the interference term. Let us now define the XNLD signal for the present experimental configuration as $\mathrm{XNLD}=\operatorname{XAS}\left(90^{\circ}\right)-\operatorname{XAS}\left(0^{\circ}\right)$ (Fig. 3c). It is dominated by a derivative signal centred around $8380 \mathrm{eV}$ that corresponds to the quadrupole XNLD. The energy difference between the maximum and the minimum $(\approx 2.3 \mathrm{eV})$ gives an estimation of the cubic crystal field splitting between empty $e_{g}$-like $5 d$ orbitals (probed at peak Q1) and empty $t_{2 g}$-like $5 d$ orbitals (probed at peak Q2) in the presence of the $2 s$ core hole, which is close to the ground state value. The positive feature at $8372 \mathrm{eV}$ originates solely from the interference term, without any contamination from other terms. It is therefore directly related to the $4 f-6 p$ mixing on Gd. Note that the shape and intensity of XNLD, especially at low energy (peak $\mathbf{O}$ ), strongly depends on the emission energy used to detect HERFD-XAS spectra. The overall shape of the calculated XNLD is in very good agreement with the experimental signal measured with $E_{e m 1}$. 
(a)

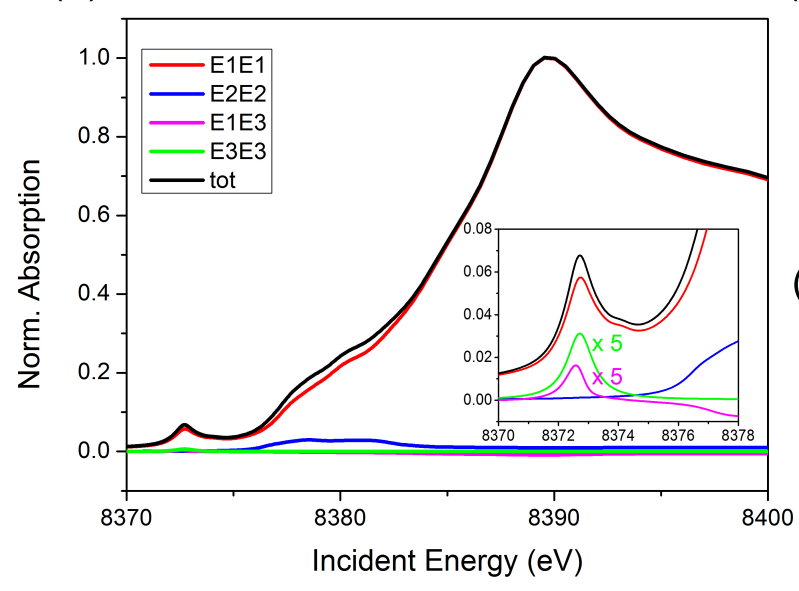

(b)

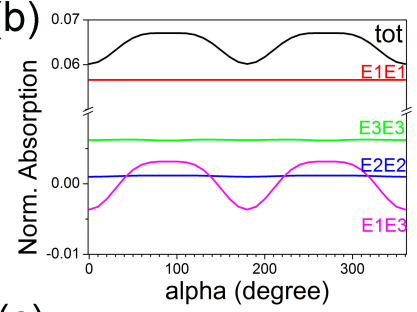

(c)

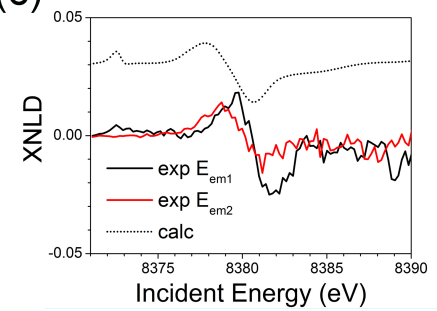

(d)

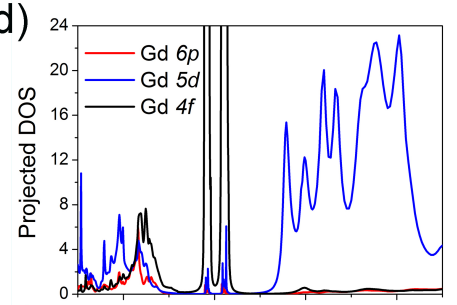

(e)

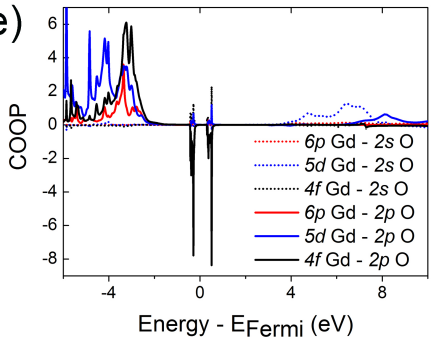

FIG. 3: (a) XAS spectrum calculated for $\alpha=90^{\circ}$ showing the different contributions. Inset: zoom in the pre-edge. (b) Contribution of the different terms to the angular dependence of peak O. (c) Experimental XNLD measured for two different values of the emission energy (solid lines) and calculated XNLD (dots). The calculation has been shifted by 0.03 in the vertical axis for clarity. (d) Gd projected partial DOS and COOP (e) (number of electrons/Ry) calculated in the ground state close to the Fermi level $(\approx 8372.8 \mathrm{eV})$.

However, slight differences in intensity and peak position are noticed, which we explain by the fact that our first-principle approach provides the XAS cross-section (not HERFD-XAS) not taking into account the emission process, and by the possible limitation of DFT in modeling localized $4 f / 5 d$ levels and of using a static core-hole description.

Quantification of $G d 4 f-6 p$ mixing and Gd-O orbital hybridization in the ground state. We have derived a new magneto-optical sum rule for the XNLD of the interference term (Suppl. Inf. II [32]). Similarly to the ones derived for XMCD [33-35] that remarkably popularized the technique, this sum rule is expressed in a simple form and can be directly applied to the experimental data. First we obtained a general expression, which then nicely simplifies by considering a cubic crystal, $L_{1}$-edge XAS and for the present single crystal orientation as:

$$
\begin{aligned}
& \Sigma_{E 1 E 3-X N L D}(\alpha)=\int \frac{\sigma_{\text {cube }}^{\text {dichro }}(E 1, E 3)(\alpha)}{(\hbar \omega)^{3}} d(\hbar \omega) \\
& =\frac{\pi \alpha_{0}}{(\hbar c)^{2}}(-19+60 \cos 2 \alpha+15 \cos 4 \alpha) D_{1} O_{3}\left\langle I\left|M_{130}^{4}\right| I\right\rangle
\end{aligned}
$$

where $D_{1}$ and $O_{3}$ are the radial dipole and octupole integrals respectively. The sum rule relates the integral of the XNLD of the interference term to the ground state hexadecapole moment $\left\langle I\left|M_{130}^{4}\right| I\right\rangle$ that fully quantifies by itself the mixing of $p$ and $f$ orbitals. The integral runs over the small energy range where empty $4 f$ states are probed $(8370-8373 \mathrm{eV})$, which makes its calculation tractable. In the general case of non-cubic crystals, $\Sigma_{E 1 E 3-X N L D}$ is expressed as a combination of several ground state moments $\left\langle I\left|M_{\ell \ell^{\prime} \gamma}^{g}\right| I\right\rangle$ where the operator $M_{\ell \ell^{\prime} \gamma}^{g}=\sum_{m m^{\prime}}(-1)^{\ell-m}\left(\ell-m \ell^{\prime}-m^{\prime} \mid g \gamma\right) a_{\ell m} a_{\ell^{\prime} m^{\prime}}^{\dagger}$ measures the mixing of $\ell$ and $\ell^{\prime}$ orbitals reached from a $\ell_{0}$ core orbital coupled at order $g$. In the case of $L_{1}$ edge $\left(\ell_{0}=0, \ell=1, \ell^{\prime}=3\right), M_{\ell \ell^{\prime} \gamma}^{g}$ is analogous to a quadrupole moment for $g=2$, an octupole moment for $g=3$ and an hexadecapole moment for $g=4$ : these moments are "cross-terms" since they couple states with same parity but with different values of angular momentum.

In order to apply the E1E3 XNLD sum rule to the experimental data, regardless of spectra normalization, we have first applied the quadrupole XNLD and XAS sum rules in the energy range where $5 d$ states are probed below the continuum (8373-8388 eV). The quadrupole XNLD sum rule (Suppl. Inf. III [32]) and the quadrupole XAS sum rule write respectively:

$$
\begin{aligned}
& \Sigma_{E 2 E 2-X N L D}(\alpha)=\int \frac{\sigma_{\text {cube }}^{\text {dichro }}(E 2, E 2)(\alpha)}{(\hbar \omega)^{3}} d(\hbar \omega) \\
& =\frac{3 \pi \alpha_{0}}{2(\hbar c)^{2}} Q_{2}^{2}(-19+60 \cos 2 \alpha+15 \cos 4 \alpha)\left\langle I\left|M_{220}^{4}\right| I\right\rangle, \\
& \Sigma_{E 2 E 2-X A S}=\int \frac{\sigma_{\text {cube }}^{\text {iso }}(E 2, E 2)}{(\hbar \omega)^{3}} d(\hbar \omega)=\frac{\pi^{2} \alpha_{0}}{(\hbar c)^{2}} Q_{2}^{2} \frac{10-n}{75},
\end{aligned}
$$

where $(10-n)$ is the number of $5 d$ holes, $Q_{2}$ the radial quadrupole integral and $M_{220}^{4}$ the (uncrossed) hexadecapole moment associated to $5 d$ orbitals. Note that the quadrupole XAS sum rule applies to the isotropic part of the quadrupole. The ratio of $\Sigma_{E 2 E 2-X N L D}(\alpha)$ and $\Sigma_{E 2 E 2-X A S}$ provides an absolute measurement of $\left\langle I\left|M_{220}^{4}\right| I\right\rangle$, which now allows the absolute determination of $\left\langle I\left|M_{130}^{4}\right| I\right\rangle$ by taking the ratio of $\left[\Sigma_{E 1 E 3-X N L D}\left(90^{\circ}\right)-\Sigma_{E 1 E 3-X N L D}\left(0^{\circ}\right)\right]$ and $\left[\Sigma_{E 2 E 2-X N L D}\left(90^{\circ}\right)-\Sigma_{E 2 E 2-X N L D}\left(0^{\circ}\right)\right]$, which is equal to $\frac{2}{3} \frac{D_{1} O_{3}}{Q_{2}^{2}} \frac{\left\langle I\left|M_{130}^{4}\right| I\right\rangle}{\left\langle I\left|M_{220}^{4}\right| I\right\rangle}$. Taking the values of the radial in- 
tegrals extracted from FDMNES (Suppl. Inf. IV [32]), application of the sum rules to the theoretical data of Fig. 3c yields: $\left\langle I\left|M_{130}^{4}\right| I\right\rangle=1 \cdot 1 \cdot 10^{-4}$ (dimensionless).

The fact that Gd $4 f$ states mix with $6 p$ states despite their localized character suggests that they may also participate to the bonding with O ligands. Further evidence is given by the projected DOS and COOP calculated close to the Fermi level for Gd in the ground state (Fig. 3d-e). Both features around $0 \mathrm{eV}$ in the DOS correspond to the localized occupied and empty Gd $4 f$ states, and the negative $\mathrm{Gd} 4 f$-O $2 p$ COOP reveals a sizeable anti-bonding character that is the dominating contribution, while the Gd $4 f$-O $2 s$ COOP shows a bonding character approx. 4 times weaker. The Gd $5 d$ states form a band spread over $7 \mathrm{eV}$, with a bonding character first with the $\mathrm{O} 2 s$ and then the $\mathrm{O} 2 p$ orbitals at higher energy.

This work provides a novel approach to measure the hybridization degree of $f$ states in rare earths by measuring original information on the electronic structure that was not accessible before. Hybridization of empty $f$ and $p$ states can be measured on any XAS beamline equipped with high resolution detection, using the interference between electric octupole and dipole transitions at the $L_{1}$ edge and then applying the sum rule to calculate the ground state $f-p$ mixing. Contrary to the electric dipole-quadrupole interference that is measured by XNCD, the electric dipole-octupole interference exists also in the presence of parity symmetry in the crystal: it is therefore a much more general effect. The recent progress achieved in brilliance for x-ray sources now offer the appropriate conditions to measure such weak dichroic effects, which we expect to further grow in importance in the coming years.

* Electronic address: amelie.juhin@upmc.fr

* Electronic address: amelie.juhin@upmc.fr

[1] M. Humphries, Rare earth elements: the global supply chain (2013).

[2] A. Kerridge, Chemical Communications 53, 6685 (2017).

[3] M. W. Loble, J. M. Keith, A. B. Altman, S. C. E. Stieber, E. R. Batista, K. S. Boland, S. D. Conradson, D. L. Clark, J. Lezama Pacheco, S. A. Kozimor, et al., Journal of the American Chemical Society 137,2506 (2015).

[4] M. L. Neidig, D. L. Clark, and R. L. Martin, Coordination Chemistry Reviews 257, 394 (2013).

[5] E. Lu, S. Sajjad, V. E. Berryman, A. J. Wooles, N. Kaltsoyannis, and S. T. Liddle, Nature communications 10, 634 (2019).

[6] F. De Groot and A. Kotani, Core level spectroscopy of solids (CRC press, 2008).

[7] C. Booth, Y. Jiang, D. Wang, J. Mitchell, P. Tobash, E. Bauer, M. Wall, P. Allen, D. Sokaras, D. Nordlund, et al., Proceedings of the National Academy of Sciences 109, 10205 (2012).

[8] H. Asakura, T. Shishido, K. Teramura, and T. Tanaka, The Journal of Physical Chemistry C 119, 8070 (2015).

[9] C. Hennig, Physical Review B 75, 035120 (2007).

[10] N. Smolentsev, A. A. Guda, O. V. Safonova, M. A. Soldatov, C. Paun, G. Smolentsev, J. A. van Bokhoven, and A. V. Soldatov, Journal of Physics: Conference Series 430, 012062 (2013).

[11] S. G. Minasian, J. M. Keith, E. R. Batista, K. S. Boland, D. L. Clark, S. D. Conradson, S. A. Kozimor, R. L. Martin, D. E. Schwarz, D. K. Shuh, et al., Journal of the American Chemical Society 134, 5586 (2012).

[12] A. Ashley, G. Balazs, A. Cowley, J. Green, C. H. Booth, and D. O'Hare, Chemical Communications pp. 1515-1517 (2007).

[13] A. B. Altman, C. D. Pemmaraju, S. Alayoglu, J. Arnold, E. D. Bauer, C. H. Booth, Z. Fisk, J. I. Pacold, D. Prendergast, D. K. Shuh, et al., Physical Review B 97, 045110 (2018).

[14] K. O. Kvashnina, S. M. Butorin, and P. Glatzel, Journal Analytical Atomic Spectrometry 26, 1265 (2011).

[15] S. M. Butorin, K. O. Kvashnina, A. L. Smith, K. Popa, and P. M. Martin, Chemistry A European Journal 22, 9693 (2016).

[16] G. Schütz, W. Wagner, W. Wilhelm, P. Kienle, R. Zeller, R. Frahm, and G. Materlik, Physical Review Letters 58, 737 (1987).

[17] H. Dürr, G. Guo, G. Van der Laan, J. Lee, G. Lauhoff, and J. Bland, Science 277, 213 (1997).

[18] B. T. Thole, G. van der Laan, and G. A. Sawatzky, Physical Review Letters 55, 2086 (1985).

[19] L. Alagna, T. Prosperi, S. Turchini, J. Goulon, A. Rogalev, C. Goulon-Ginet, C. R. Natoli, R. D. Peacock, and B. Stewart, Physical Review Letters 80, 4799 (1998).

[20] C. Brouder, Journal of Physics: Condensed Matter 2, 701 (1990).

[21] S. M. Butorin, K. O. Kvashnina, J. R. Vegelius, D. Meyer, and D. K. Shuh, Proceedings of the National Academy of Sciences 113, 8093 (2016).

[22] P. Glatzel and A. Juhin, X-ray Absorption and Emission Spectroscopy (John Wiley and Sons, Ltd, 2013), chap. 2, pp. 89-171, ISBN 9781118681909.

[23] S. Bernadotte, A. J. Atkins, and C. R. Jacob, The Journal of Chemical Physics 137, 204106 (2012).

[24] R. F. Pettifer, S. P. Collins, and D. Laundy, Nature 454, 196 (2008).

[25] O. Bunău and Y. Joly, Physical Review B 85, 155121 (2012).

[26] H. Sawada, Journal of Solid State Chemistry 132, 300 (1997).

[27] R. Dronskowski and P. E. Blöchl, The Journal of Physical Chemistry 97, 8617 (1993).

[28] N. Bouldi, N. J. Vollmers, C. G. Delpy-Laplanche, Y. Joly, A. Juhin, P. Sainctavit, C. Brouder, M. Calandra, L. Paulatto, F. Mauri, et al., Physical Review B 96, 085123 (2017). 
[29] C. Brouder, A. Juhin, A. Bordage, and M.-A. Arrio, Journal of Physics: Condensed Matter. 20, 455205 (2008).

[30] D. Cabaret, C. Brouder, M.-A. Arrio, P. Sainctavit, Y. Joly, A. Rogalev, and J. Goulon, Journal of Synchrotron Radiation 8, 460 (2001).

[31] A. Juhin, C. Brouder, M.-A. Arrio, D. Cabaret, P. Sainctavit, E. Balan, A. Bordage, A. P. Seitsonen, G. Calas, S. G. Eeckhout, et al., Physical Review B 78, 195103 (2008).

[32] See supplemental material at [url will be inserted by publisher] for the derivation and application of the dipole-octupole and quadrupole-quadrupole sum rules.
[33] B. T. Thole, P. Carra, F. Sette, and G. van der Laan, Physical Review Letters 68, 1943 (1992).

[34] P. Carra, H. König, B. T. Thole, and M. Altarelli, Physica B 192, 182 (1993)

[35] C. T. Chen, Y. U. Idzerda, H.-J. Lin, N. V. Smith, G. Meigs, E. Chaban, G. H. Ho, E. Pellegrin, and F. Sette, Physical Review Letters 75, 152 (1995).

[36] The ratio of the magnetic to electric dipole contributions to the XAS cross-section is $10^{-5}$ for Hf at the $L_{1}$ edge.

[37] For the sake of simplicity the notation $\sigma(E m, E m)$ $(m \neq n)$ gathers $|\langle F|E m| I\rangle| \cdot\left|\left\langle I\left|E n^{*}\right| F\right\rangle\right|$ and its complex conjugate 